\title{
The fMRI BOLD signal tracks electrophysiological spectral perturbations, not event-related potentials
}

\author{
Andrew D. Engell a, Scott Huettel ${ }^{\mathrm{b}}$, Gregory McCarthy ${ }^{\mathrm{a}, *}$ \\ a Human Neuroscience Laboratory, Department of Psychology, Yale University, New Haven, CT, USA \\ ${ }^{b}$ Center for Interdisciplinary Decision Sciences, Psychology and Neuroscience Department, Duke University, Durham, NC, USA
}

\section{A R T I C L E I N F O}

Article history:

Received 28 June 2011

Revised 3 August 2011

Accepted 25 August 2011

Available online 8 September 2011

\section{Keywords:}

EEG

ECoG

ERP

Gamma

Hemodynamic

\begin{abstract}
A B S T R A C T
Functional magnetic resonance imaging (fMRI) and electroencephalography (EEG) are primary tools of the psychological neurosciences. It is therefore important to understand the relationship between hemodynamic and electrophysiological responses. An early study by Huettel and colleagues found that the coupling of fMRI blood-oxygen-level-dependent signal (BOLD) and subdurally-recorded signal-averaged event-related potentials (ERPs) was not consistent across brain regions. Instead, a growing body of evidence now indicates that hemodynamic changes measured by fMRI reflect non-phase-locked changes in high frequency power rather than the phase-locked ERP. Here, we revisit the data from Huettel and colleagues and measure event-related spectral perturbations (ERSPs) to examine the time course of frequency changes. We found that, unlike the ERP, $\gamma$-ERSP power was consistently coupled with the hemodynamic response across three visual cortical regions. Stimulus duration modulated the BOLD signal and the $\gamma$-ERSP in the peri-calcarine and fusiform cortices, whereas there was no such modulation of either physiological signal in the lateral temporal-occipital cortex. This finding reconciles the original report with the more recent literature and demonstrates that the ERP and ERSP reflect dissociable aspects of neural activity.
\end{abstract}

(c) 2011 Elsevier Inc. All rights reserved.

\section{Introduction}

Psychological neuroscientific studies predominantly rely on functional magnetic resonance imaging (fMRI) and electroencephalography (EEG) to provide insight into the neural processes that support perception, cognition, and sensorimotor processing. Each of these methods provides a distinct, although comparable, measurement of the location and latency of task-associated neural signals. fMRI measures the blood-oxygen-level-dependent (BOLD) signal to estimate hemodynamic changes, which arise from the metabolic demands of neural activity (e.g., Hoge et al., 1999). EEG measures local field potentials (LFPs) generated by the aggregation of post-synaptic potentials from neuronal assemblies (i.e., integrative activity associated with inputs to a region). Because the physiological activity that engenders hemodynamic responses might not necessarily also give rise to measurable LFPs, and vice versa, understanding the nature of neurovascular coupling reflected in the BOLD signal has been a core goal for neuroimaging research.

In a seminal study, Logothetis et al. (2001) simultaneously measured BOLD changes, LFPs, and multi-unit activity (MUA) from macaque visual cortex.

\footnotetext{
* Corresponding author at: Psychology, PO Box 208205, New Haven, CT 06520-8205, USA. Fax: + 12034327172 .

E-mail address: gregory.mccarthy@yale.edu (G. McCarthy).
}

LPFs and MUA were both found to be coupled with hemodynamic changes, but LFPs were the best predictors of the amplitude and time course of the BOLD signal. These results were the first to demonstrate a direct link between BOLD signal changes and neural activity. However, the focus of this initial investigation was limited to primary visual cortex, leaving open the question of whether this neurovascular relationship was stable across brain regions.

Huettel et al. (2004) explicitly tested the stability of this relationship in the human brain by examining three separate cortical regions of interest (ROIs) - peri-calcarine cortex (pC), fusiform gyrus (FG), and lateral-temporal-occipital (LTO) cortex - while participants viewed static visual stimuli for varying durations. EEG was acquired from electrodes placed subdurally on the cortical surface of epilepsy patients being evaluated for possible cortical resection. The duration sensitivity of event-related potentials (ERPs), phase-locked changes in the EEG, was compared to that of the BOLD signal. Consistent with the findings of Logothetis et al. (2001), the electrophysiological and hemodynamic measures were both modulated by stimulus duration within the primary visual cortex. However, the critical examination of ROIs outside of primary visual cortex found that BOLD-ERP correspondence was not present within all regions. Within the LTO, ERPs and the BOLD signal showed similar duration sensitivity in that neither measure was modulated by duration. Yet, in the FG the measures were not coupled: increasing stimulus duration robustly modulated the BOLD signal but had minimal effects on the amplitude and duration of ERP components. 
The inconsistent covariation between BOLD and ERP signals might indicate regional variation in neurovascular coupling, or any of several other possible explanations. As Huettel et al. (2004) speculated, the weaker relationship in the FG might have been due to the spatial resolution of the two methods. The spatial extent of hemodynamic changes extends beyond the underlying neural response (Malonek and Grinvald, 1996). Thus, BOLD changes may exist where there is no concomitant neural, and therefore, electrical activity. Similarly, due to volume conduction of electrical fields, changes in the ERP can reflect activity from distant neural generators. This possibility is exacerbated by signal averaging, in which the contribution of nonstimulus-locked local neural activity is greatly diminished in favor of stimulus-locked activity that may be local or distant. It is therefore possible that the spatial imprecision of these techniques gave rise to the absence of covariation within the FG. Or, as a more intriguing possibility, the BOLD response may be coupled to aspects of neuronal activity that do not themselves modulate the ERP signal.

Since the publication of these early studies, the nature of the relationship between BOLD changes and neural activity has remained a topic of great interest. In recent years, many investigations of neurovascular coupling in the brains of human and non-human animals have demonstrated a tight coupling of changes in $\gamma$-band power and the BOLD signal (Fisch et al., 2009; Goense and Logothetis, 2008; Jerbi et al., 2009; Kayser et al., 2004; Koch et al., 2009; Lachaux et al., 2007; Mukamel et al., 2005; Niessing et al., 2005; Ojemann et al., 2010; Sirotin and Das, 2009; Viswanathan and Freeman, 2007). Collectively, these investigations have probed the response in multiple brain regions, suggesting that this coupling generalizes beyond visual cortex. Further, we have previously shown that the functional response properties of the BOLD signal are shared by high-frequency oscillations in the $\gamma$-band, but not the ERP (Engell and McCarthy, 2010, 2011).

In light of this evidence, we hypothesize that, despite the inconsistent coupling of BOLD and ERP, coupling of $\gamma$-band power and BOLD is conserved across brain regions. Here we revisit the data of Huettel et al. (2004) to test this hypothesis by focusing our analysis on event-related spectral perturbations (ERSPs). Unlike ERPs, ERSPs do not depend on a phase-locked relationship with the instigating stimulus. Rather, ERSPs reflect stimulus-induced changes in spectral power within particular frequency bands. We predict ROIs that show BOLD sensitivity to stimulus-duration will also show $\gamma$-band ERSP sensitivity, thus demonstrating coupling of hemodynamic and high-frequency responses across brain regions.

\section{Materials and methods}

The description of the experimental design and data acquisition is adapted from the original report by Huettel et al. (2004).

\section{Experimental design}

This study used similar versions of the same task in an fMRI experiment and in a separate EEG experiment. The former relied on the participation of neurotypical volunteers, whereas the latter relied on the participation of epilepsy patients being monitored for possible surgery.

On each trial, a single high-contrast black-and-white radial checkerboard was presented at fixation for one of three durations: 100, 500 , or $1500 \mathrm{~ms}$. The radial checkerboard had maximum spatial frequency near the fovea ( $\sim 4$ cycles/degree, at $1^{\circ}$ from fixation) and spatial frequency decreased linearly to its minimum at the edges of the display ( $\sim 0.25$ cycles/degree, at $10^{\circ}$ from fixation). The stimulus did not cycle on and off, but remained static over its duration to facilitate ERP recording of onset and offset potentials. In the fMRI experiment, stimuli were projected into the scanner bore onto a screen, which the subject viewed using mirrored goggles. The resulting field of view subtended $\sim 20^{\circ}-15^{\circ}$ of visual angle. In the ERP experiment, subjects were in a hospital bed and viewed the stimuli on an LCD computer monitor, whose display subtended $\sim 18^{\circ} \mathrm{x} 14^{\circ}$ of visual angle.

In both experiments, the interstimulus interval (ISI) was sufficiently long to preclude refractory effects from preceding trials. The mean ISI for the fMRI experiment was $16 \mathrm{~s}$ (range 15-17 s) and the mean ISI for the ERP experiment was $6 \mathrm{~s}$ (range 5-7 s). All stimuli were presented using the CIGAL display environment (Voyvodic, 1999). All fMRI participants viewed a total of 220 stimuli. Eight ERP participants viewed a total of 126 stimuli over three runs that were separated by rest periods. One ERP participant viewed 84 stimuli over two runs. For both fMRI and ERP studies, the three stimulus durations were randomly ordered within each run.

\section{fMRI image acquisition and analysis}

Twelve young adults (seven females, five males, mean age 21 years) participated in the fMRI study, which was conducted at the Duke-UNC Brain Imaging and Analysis Center and approved by the Duke IRB. All MR images were acquired using a 4.0 T GE NVI scanner. To ensure coverage of the ROIs, eight 5 -mm thick slices were acquired parallel to the anterior-posterior commissure line, chosen to encompass $\mathrm{pC}$ cortex and FG. T1-weighted spin-echo images were acquired at each slice location for identification of anatomically based regions of interest (in-plane resolution $=0.9375 \mathrm{~mm}^{2}$ ). For functional imaging, we acquired $\mathrm{T} 2{ }^{*}$-weighted images sensitive to BOLD contrast using a spiral-out gradient-echo pulse sequence $(T R=500 \mathrm{~ms}$, $\mathrm{TE}=20 \mathrm{~ms}$, flip angle $=20^{\circ}$, in-plane resolution $=3.75 \mathrm{~mm}^{2}$ ).

For each participant, the $\mathrm{pC}$ and FG were delineated based on anatomical landmarks. Due to the difficulty of determining anatomical boundaries for V5, we defined the LTO ROIs by identifying the voxel with maximal significance and then selecting all contiguous active voxels. The mean BOLD signal, extending from $5 \mathrm{~s}$ prior- to $12 \mathrm{~s}$ post-stimulus onset, was extracted from active voxels within the three ROIs. Significance at each voxel was determined by correlating the mean response with a canonical HRF.

\section{Intracranial EEG acquisition}

Recordings were obtained from nine patients (five females, four males, mean age 31 years) with medically intractable epilepsy who were being evaluated for possible surgery by the Yale Epilepsy Surgery Program (Spencer et al., 1982). In these patients, strips or grids of stainless steel electrodes ( $2.2 \mathrm{~mm}$ in surface diameter) were placed subdurally on the cortical surface. The placement of the strips was determined by the clinical needs of each patient, and thus electrode locations varied across individuals. The study reported here was among several sensory and cognitive intracranial EEG experiments in which each subject participated, typically 4-8 days following implantation of electrodes. At the time of participation, medication levels to control seizures and post-operative pain varied across patients. The EEG experiments were not conducted immediately before or after seizures nor were any of our sites of interest revealed to be in epileptogenic cortex. The EEG protocol was approved by the IRB of the Yale University School of Medicine. All participants provided informed consent.

Local field potentials were recorded simultaneously from 128 electrode sites and amplified with a common mastoid reference using an SA Instruments EEG amplifier system with a $0.1-100 \mathrm{~Hz}$ bandpass. The EEG signal was continuously acquired and digitized with 14-bit resolution using a Microstar 4200 A/D data acquisition board. The digitized signal was sampled at $250 \mathrm{~Hz}$ and written to disk using a custom PC-based acquisition system. A digital code unique to each experimental condition was recorded in a separate channel at the onset of each stimulus presentation. 
Electrodes of interest were localized within the $\mathrm{pC}$ (35 electrodes from 8 of 9 patients), FG (17 electrodes from 8 of 9 patients), and LTO (22 electrodes from 9 of 9 patients).

\section{Event-related potential (ERP) analysis}

ERP analyses were performed using custom MATLAB (The Mathworks, Inc.) functions. Residual line noise $(60-\mathrm{Hz})$ filtering was performed in Matlab using a 5th order Butterworth filter that was applied in a temporally symmetric manner to avoid introducing phase shifts. Baseline adjusted ERPs were created by signal averaging the EEG signal across trials for each experimental condition and subtracting from each time point the average of a $200 \mathrm{~ms}$ pre-stimulus epoch. A temporally symmetric smoothing kernel with a total length of 13 time-points (from -6 to +6 time points) was convolved with the average ERP waveforms prior to plotting.

\section{Event-related spectral perturbation (ERSP) analysis}

We removed the mean (unsmoothed) signal-averaged ERP from the raw EEG signal for each trial prior to frequency decomposition. This ensured that any significant ERSP differences between conditions did not merely reflect the frequency composition of the phase-locked ERP. As a result of this approach, the frequency-domain analysis reported here is insensitive to spectral changes that undergo phase resetting (i.e., phase-locked 'evoked' EEG responses). However, these spectra are captured in the time-domain analysis (i.e., ERP), resulting in a full characterization of the EEG data. Time-frequency analysis in which the mean response was not removed was also performed. These results only differed in that the frequency domain representation of the ERPs was evident, but did not reveal any additional duration dependent effects and will not be discussed further.

ERSPs were computed using EEGLAB v7.1 (Delorme and Makeig, 2004) and MATLAB v7.9 (The Mathworks, Inc.). Time-frequency power spectra were estimated using Morelet wavelet analysis based on 3 cycles at the lowest frequency $(9 \mathrm{~Hz}$ ) increasing to 20.75 cycles at the highest frequency $(125 \mathrm{~Hz})$. Change in power induced by each category (i.e., ERSP) was estimated by calculating the ratio of logpower $(\mathrm{db})$ between the post-stimulus $(0-1800 \mathrm{~ms})$ and pre-stimulus ( $-800-0$ ms ) epochs. ERSPs within the $\alpha$-band (9-12 Hz), the $\beta$-band $(12-30 \mathrm{~Hz})$, and the $\gamma$-band $(30-100 \mathrm{~Hz})$ were averaged at each timepoint to create a 'power-wave' over time. The amplifiers used in our studies imposed a $100 \mathrm{~Hz}(-3 \mathrm{~dB})$ upper limit on the EEG signal, and so we restricted the upper range of the $\gamma$-band to $100 \mathrm{~Hz}$.

ERSP differences were tested using paired $t$-tests of area under the curve (AUC) estimates of the power-waves. AUC was estimated within two temporal windows (500-1000 ms and 1000-1500 ms) to best capture duration dependent differences across conditions. Statistical analysis of the $100 \mathrm{~ms}$ condition (contrasted with the 500 and $1500 \mathrm{~ms}$ conditions) was performed using the AUC estimates from the 500$1000 \mathrm{~ms}$ epoch. Statistical analysis of the difference between the 500 and 1500 ms conditions was performed using the AUC estimates from the 1000-1500 ms epoch. All contrasts were tested using paired $t$-tests.

\section{Results}

Peri-calcarine cortex $(p C)$

$\gamma$-ERSP

The $\gamma$-ERSP was significantly modulated by stimulus duration (Figs. 1 and 2). The response to the $100 \mathrm{~ms}$ condition was smaller than both the $500 \mathrm{~ms}(t(34)=-10.70, p<.001)$ and $1500 \mathrm{~ms}(t(34)=$ $-11.79, p<.001)$ conditions. The 500 ms condition was smaller than the 1500 ms condition $(t(34)=-10.99, p<.001)$. Across all conditions, an initial peak was followed by a duration-dependent increase that was almost as large as the onset peak (Fig. 2, column 1; row 5).

\section{$\beta$-ERSP}

$\beta$-ERSP also showed stimulus duration dependence (Figs. 1 and 2). The $100 \mathrm{~ms}$ condition was smaller than both the $500 \mathrm{~ms}(t(34)=$ $-6.73, p<.001)$ and $1500 \mathrm{~ms}(t(34)=-5.26, p<.001)$ conditions. The $500 \mathrm{~ms}$ condition was smaller than the $1500 \mathrm{~ms}$ condition $(t(34)=$ $-3.81, p<.001)$. The $\beta$-ERSP power-waves show an initial onset peak, followed by a modest sustained response and then an offset peak (Fig. 2, column 1; row 4). Unlike the $\gamma$-ERSP, the $\beta$-ERSP revealed notable offset peaks and a smaller sustained response relative to the onset peaks.

\section{$\alpha-E R S P$}

There was no effect of stimulus duration on $\alpha$-ERSP at sites (all ps>.01) (Figs. 1 and 2).

\section{ERP}

The ERP was modulated by stimulus duration. An initial evoked response was followed by a duration dependent offset potential. Importantly, there was also a period of sustained activity between the onset and offset potentials that was observable in the 500 and 1500 ms conditions (see Huettel et al., 2004 for details) (Fig. 2, column 1; row 2).

\section{$B O L D$}

The BOLD response was modulated by stimulus duration. The amplitude and latency of the peak response increased with longer durations (see Huettel et al., 2004 for details) (Fig. 2, column 1; row 1).

Fusiform gyrus (FG)

\section{$\gamma$-ERSP}

The $\gamma$-ERSP showed a duration dependent increase in power (Figs. 1 and 2). The response to the $100 \mathrm{~ms}$ condition was smaller than both the $500 \mathrm{~ms}(t(16)=-2.22, p=.041)$ and $1500 \mathrm{~ms} \quad(t(16)=-3.32$, $p=.004)$ conditions. The $500 \mathrm{~ms}$ condition was smaller than the 1500 ms condition $(t(16)=-3.52, p<.001)$. As with the $\mathrm{pC}$, the sustained increase in $\gamma$-band power at FG sites was only marginally smaller than the initial onset peak. This is particularly evident in the $1500 \mathrm{~ms}$ condition (Fig. 2, column 2; row 5).

\section{$\beta$-ERSP}

A small effect of stimulus duration was observed in the $\beta$-ERSP. An initial duration invariant increase in $\beta$-power was followed by a seemingly duration dependent decrease in power (Figs. 1 and 2). However, only the 100 vs. 500 ms contrast was statistically significant $(t(16)=4.91, p<.001)$. Neither the 100 vs. 1500 ms contrast $(t(16)=$ $1.58, p=.134)$, nor the 500 vs. $1500 \mathrm{~ms}$ contrast $(t(16)=1.96$, $p=.068$ ) reached significance. It is likely that the lack of statistical significance is a function of the frequency band boundaries, as the $12-30 \mathrm{~Hz}$ range seems to combine a loss of power in the 'lower' beta range with an increase of power in the 'higher' beta range that abuts the border between beta and gamma (see Fig. 1, column 2).

\section{$\alpha-E R S P$}

The $\alpha$-ERSP showed a significant decrease as a function of stimulus duration (Figs. 1 and 2). Therefore, larger AUC values reflect a greater loss of alpha power. The response to the $100 \mathrm{~ms}$ condition was smaller than both the $500 \mathrm{~ms}(t(16)=5.27, p<.001)$ and $1500 \mathrm{~ms}(t(16)=$ $3.85, p<.001)$ conditions. The $500 \mathrm{~ms}$ condition was smaller than the 1500 ms condition $(t(16)=6.77, p<.001)$.

\section{ERP}

The ERP from sites within FG differed from those within $\mathrm{pC}$ in that there were no notable offset potentials or sustained activity following an initial onset potential (see Huettel et al., 2004 for details) (Fig. 2, column 2; row 2). 


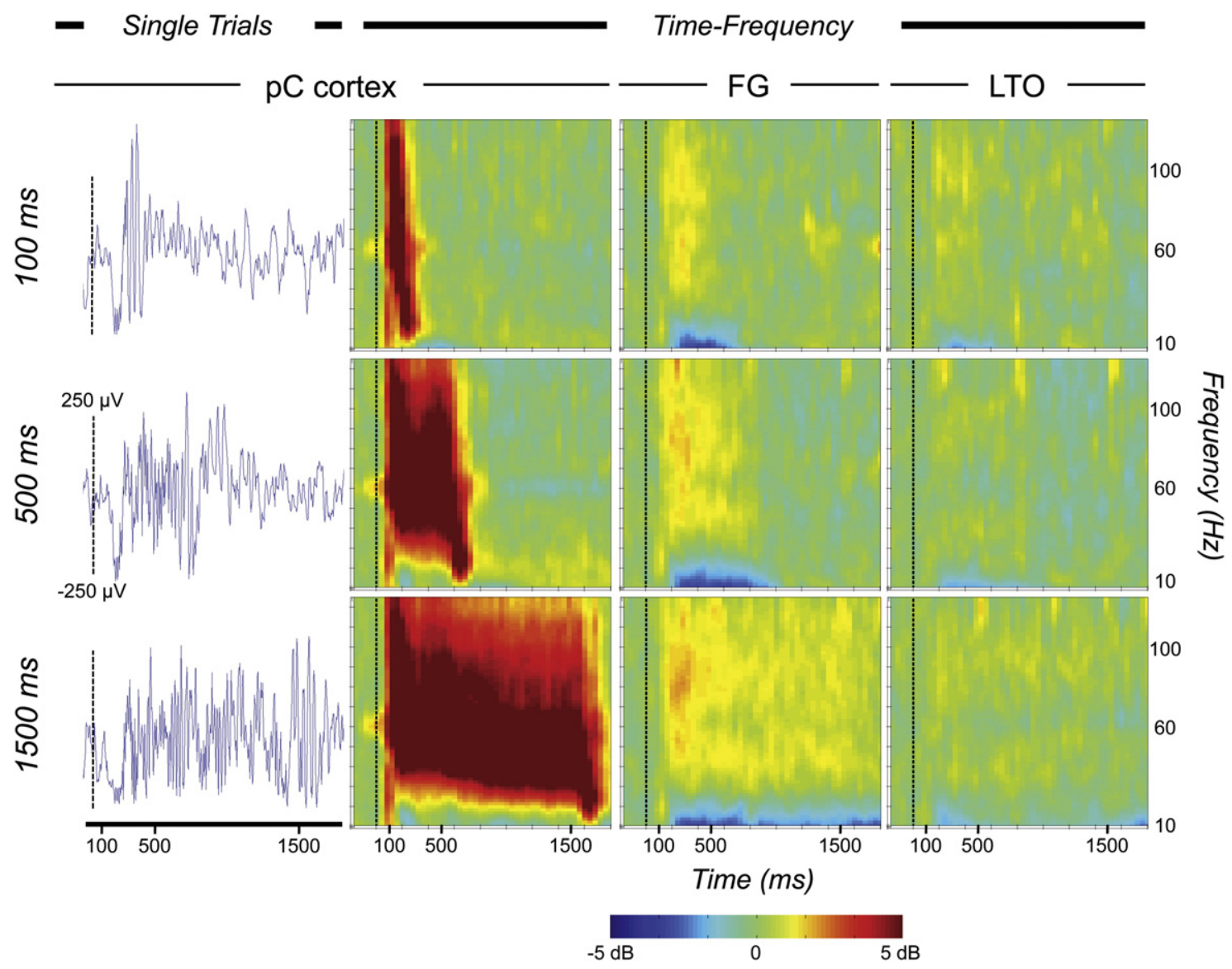

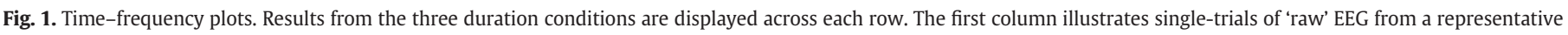

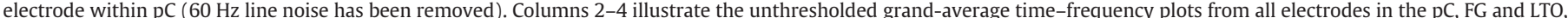

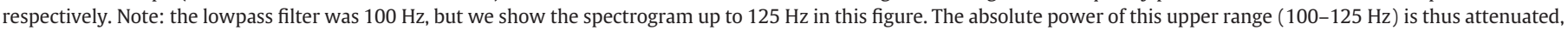
but one can still measure the relative difference in post- and pre-stimulus power.

$B O L D$

The BOLD response was modulated by stimulus duration. The amplitude and latency of the peak response increased with longer durations (see Huettel et al., 2004 for details) (Fig. 2, column 2; row 1).

LTO

\section{$\gamma$-ERSP}

There was no clear effect of stimulus duration on the $\gamma$-ERSP (Figs. 1 and 2). Neither the 100 vs. 500 ms or the 100 vs. $1500 \mathrm{~ms}$ contrasts were significantly different ( $p s>.01$ ). The 500 vs. $1500 \mathrm{~ms}$ contrast did, however, reach significance $(t(21)=-2.85, p=.01)$. Though statistically significant, it is unlikely that this is a 'real' increase as the effect is driven by a small but sustained loss in $\gamma$ power for the $500 \mathrm{~ms}$ condition between $\sim 1000$ and $1500 \mathrm{~ms}$ (Fig. 2, column 3; row 5). Furthermore, this loss of power begins at $\sim 400 \mathrm{~ms}$ after stimulus offset, suggesting that it is not an effect of the stimulus, but rather noise.

\section{$\beta$-ERSP}

There was no effect of stimulus duration on the $\beta$-ERSP (Figs. 1 and 2). No pairwise contrasts showed significant differences (all ps>.01).

$\alpha$-ERSP

There was no effect of stimulus duration on the $\alpha$-ERSP (Figs. 1 and 2). No pairwise contrasts showed significant differences (all ps>.01). However, visual inspection of the power-waves reveals a modest effect of stimulus duration with longer durations resulting in longer periods of decreased $\alpha$-power (see Fig. 2, column 3; row 3).

\section{ERP}

The ERP recorded from electrodes within LTO was similar to the ERP recorded form FG sites. After an initial transient response there were no notable offset potentials or sustained responses (Fig. 2, column 3; row 2).

\section{BOLD}

There was an increased BOLD response across all conditions, but inconsistent effects of stimulus duration (Fig. 2, column 3; row 1). There was no difference between the 100 and $500 \mathrm{~ms}$ conditions, whereas there was a small, but significant, difference between each of these and the $1500 \mathrm{~ms}$ condition (see Huettel et al., 2004 for details).

\section{Discussion}

Our results demonstrate that the relationship of the hemodynamic response with subdurally recorded EEG varies across brain regions. The BOLD signal responds to stimulus manipulations - here, visual stimuli of different duration - in a manner similar to that of high frequency components of the EEG (i.e., the $\gamma$-band) but different to that of low frequency components of the EEG (including the phase-locked ERP). Specifically, we find that both the BOLD response and $\gamma$-ERSP 

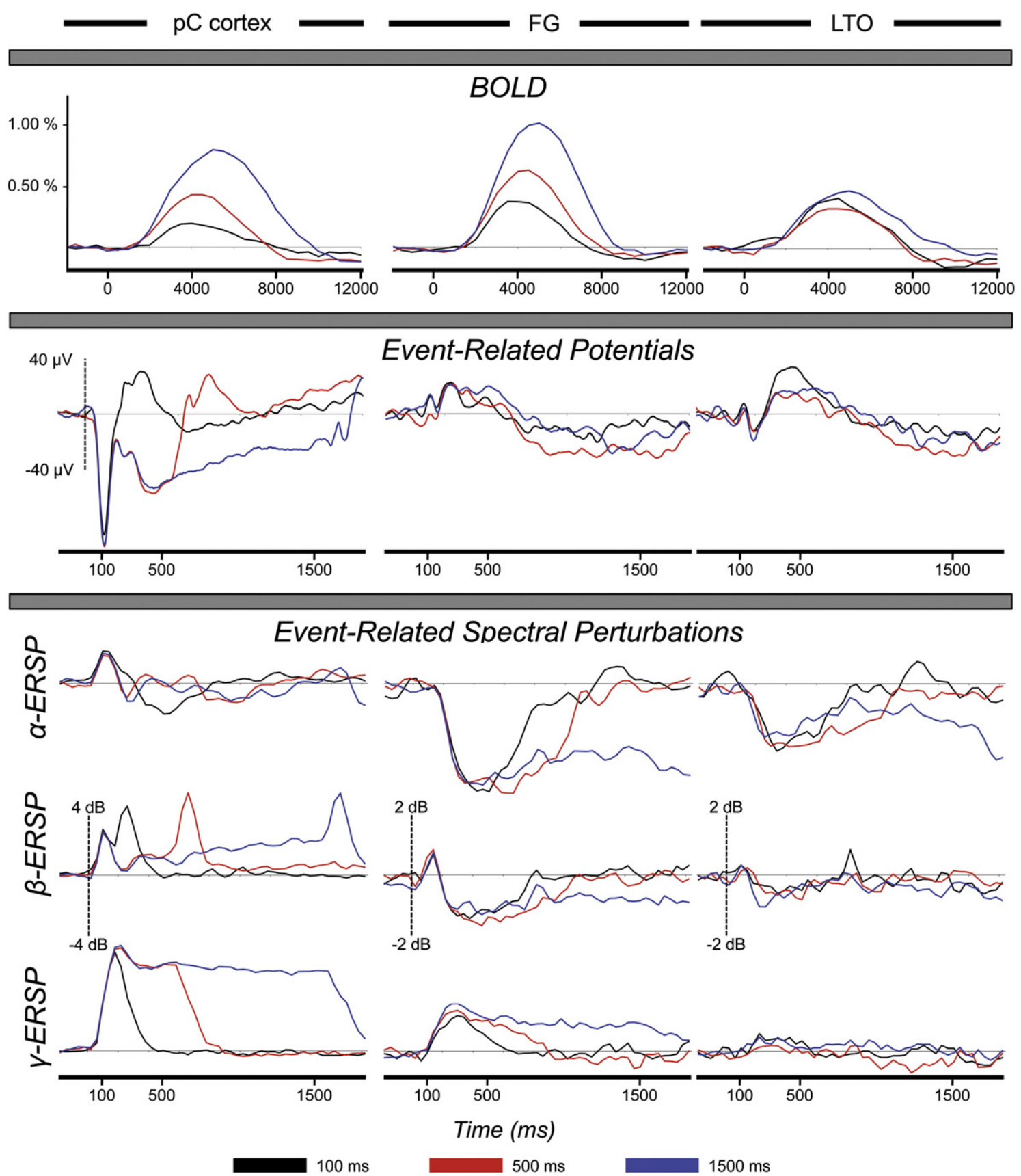

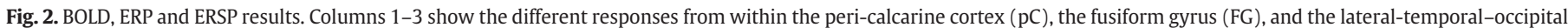

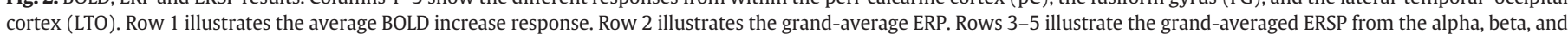
gamma frequency bands, respectively. Note: the y-axis of the ERSP plots for the pC (column 1, rows 3-5) is less sensitive than for the FG and LTO (columns 2-3, rows 3-5).

are duration-dependent across all durations in $\mathrm{pC}$ and FG, but not LTO. The $\beta$-ERSP, $\alpha$-ERSP, and ERP all show some degree of sensitivity to the stimulus duration. However, the sensitivity to stimulus duration and its relationship to the BOLD response are inconsistent across brain regions.

\section{$\gamma$-ERSP and BOLD}

Across all three ROIs there is a consistent relationship between $\gamma$ power and the BOLD response. Both the $\mathrm{PC}$ and FG show stimulus duration dependent increases in both BOLD signal and $\gamma$-ERSP. In the LTO, the BOLD and $\gamma$-ERSP effects are small and primarily driven by the $1500 \mathrm{~ms}$ stimulus duration. The similarity of these responses suggests a relationship between BOLD and $\gamma$-power that is not reflected in other components of the EEG, such as low-frequency oscillations $(<30 \mathrm{~Hz})$ or the phase-locked ERP (see below). This relationship is consistent with prior work that has demonstrated a tight coupling of high-frequency power and hemodynamic activity (Engell and McCarthy, 2010, 2011; Jerbi et al., 2009; Koch et al., 2009; Lachaux et al., 2007; Mukamel et al., 2005; Niessing et al., 2005; Ojemann et al., 2010; Sirotin and Das, 2009).

Despite the overall similarity between the BOLD and $\gamma$-ERSP responses, there is a notable difference in the effect size of these signals across regions. The BOLD response in the FG is approximately onethird larger than that found in the pC (see Huettel et al., 2004). We would therefore predict a similar pattern in the $\gamma$-ERSP. However, this is not the case. The increase in $\gamma$-power in the $\mathrm{pC}$ is substantially larger than that in the FG (see Fig. 2). What might account for 
this discrepancy? One possibility is that $\gamma$-power only partially accounts for the neural processes that generate the BOLD signal. A study of human primary visual cortex found that stimuli of varying spatial frequency had a differential impact on BOLD and magnetoencephalography $\gamma$-power (Muthukumaraswamy and Singh, 2009). Both physiological signals increased in the presence of the visual stimulus, but only the $\gamma$-power showed an additional sensitivity to spatial frequency.

Alternatively, this difference might be due to greater functional and anatomical homogeneity in the $\mathrm{pC}$ than in the FG. The functional properties of the primary visual system (e.g., V1 and V2) are reasonably well defined by the local anatomy of posterior occipital cortex (DeYoe et al., 1996; Engel et al., 1997; Sereno et al., 1995), whereas functionally defined regions along the FG are known to be anatomically variable across individuals (cf., Puce et al., 1995). Visual inspection of the response at each electrode within each ROI shows varying proportions of sites at which we noted a qualitative effect of stimulus duration on the duration of increased $\gamma$ power; $94.3 \%$ (33 of 35) in pCal, $41.2 \%$ ( 7 of 17 ) of FG sites and 9\% ( 2 of 22 ) of LTO sites. This variation of the percentage of 'sensitive' electrodes within each region lends support to the notion that greater heterogeneity within the FG accounts for some of the difference in size of the average response. Moreover, this heterogeneity would be less apparent in hemodynamic imaging than in the electrophysiology. The BOLD signal likely extends spatially beyond the region of localization with neural activity (Malonek and Grinvald, 1996), whereas high-frequency intracranial LFPs are known to rapidly decay as a function of distance from the neural generator (cf., Jerbi et al., 2009).

No consistent effect of stimulus duration (i.e., change as a function of duration across all conditions) is observed in any of the physiological measures at LTO, however the BOLD signal does show an effect of stimulus presentation. If the BOLD response largely reflects $\gamma$-power, why is there no $\gamma$-ERSP increase in the LTO? One possibility lies in the differential specificity of the two measures. The LTO ROI was functionally defined in the imaging study and anatomically defined in the EEG study. The imaging study was thus guaranteed to emphasize the contribution of stimulus-sensitive sub-regions within the LTO, regardless of their sparseness. Conversely, the anatomically defined EEG ROI includes all recording sites regardless of stimulus sensitivity and, therefore, would be less likely to reveal a $\gamma$-ERSP increase in the ROI average.

\section{$\beta-E R S P$ and $B O L D$}

The $\beta$-ERSP also shows duration dependence in the $\mathrm{pC}$ and FG. Unlike the $\gamma$-ERSP, however, the direction of the power change is not consistent across these regions. Within $\mathrm{pC}$ there is an initial spike in $\beta$-power at stimulus onset, followed by a sustained increase, and finally a second spike after stimulus offset.

Within the FG there is a non-significant trend towards stimulus duration dependence of the $\beta$-ERSP (see Fig. 2, column 2; row 4). Coupled with the stimulus duration dependence in the $\mathrm{pC}$, this seemingly implies a relationship between $\beta$-power and the BOLD signal. However, unlike the $\gamma$-ERSP, the $\beta$-ERSP is positive in the $\mathrm{pC}$ and negative in the FG.

\section{$\alpha$-ERSP and BOLD}

Within the FG $\alpha$-power decreases upon stimulus onset and remains suppressed relative to baseline until after stimulus offset. Low-frequency oscillations have been found to be inversely correlated with the BOLD response in the auditory (e.g., Mukamel et al., 2005), motor (e.g., Ritter et al., 2009), and visual (e.g., Goldman et al., 2002; Moosmann et al., 2003) cortices. Thus, the decrease in alpha power might also be associated with an increased BOLD response. While contribution from $\alpha$-oscillations cannot be ruled out, gamma is the only frequency band that shares the stimulus duration dependence of BOLD across all ROIs.

\section{ERP and BOLD}

As reported in Huettel et al. (2004), the ERPs recorded from the cortical surface reveal an inconsistent relationship with the BOLD signal. In the $\mathrm{pC}$, the BOLD and ERP both demonstrate sensitivity to stimulus duration. In the LTO, there is no effect of stimulus-duration in either the BOLD or ERP. In the FG, however, the BOLD response is duration dependent whereas the ERP is not.

\section{Patients and healthy controls}

A potential limitation of this study is systematic differences that might exist between epilepsy patients with neurotypical subjects. However, as discussed in Huettel et al. (2004), we believe that such differences do not account for the current findings. Durationdependent stimulus-evoked responses were found in similar regions in both groups, and further, varied as a function of ROI and electrophysiological measure in the patient group. This complex pattern of results means that any systematic differences as a result of pathology or medication in the patient group would need to selectively manifest within parts of the EEG signal (e.g., the ERP, but not the ERSP) and be further limited to only a subset of the tested ROIs. This seems unlikely, and we thus do not consider this a feasible explanation for the results.

\section{Conclusions}

The current findings extend, clarify, and revise inferences drawn in a previous report on these data. Huettel et al. (2004) first reported the absence of regional covariation between the human ERP and BOLD signal. Consistent with prior evidence, the hemodynamic and electrophysiological signals demonstrate similar responses within early visual cortex (Logothetis et al., 2001), but these similarities are not found in the FG and lateral-temporal occipital cortices. In addition to investigating the ERP, Huettel and colleagues also investigated the duration dependence of non-phase-locked oscillations and reported "... clear duration-related differences in the spectra obtained from electrodes in calcarine and fusiform cortices" (2004, p. 170). However, it was concluded that these effects were not consistent across regions. An increase in $\gamma$-power is observed in the $\mathrm{pC}$, whereas no such increase is observed in the FG. Conversely, a loss of alpha power is observed in the FG, whereas no such decrease is observed in the pC. Why does the current re-analysis of the same data lead us to qualitatively different inferences about BOLD-EEG correspondence?

First, the initial report compared the autospectra in a $2100 \mathrm{~ms}$ post-stimulus epoch to the autospectra in a $2100 \mathrm{~ms}$ pre-stimulus epoch. In the current report we used wavelet decomposition with a sliding window over time. The latter technique yields better temporal resolution and is therefore more likely to reveal short-lived changes in the post-stimulus epoch. For instance, the prior report found no pre- vs. post-stimulus onset difference in $\gamma$-power in the FG, particularly for the 100 and 500 ms conditions. Inspection of the time-frequency plots of the FG electrodes (Fig. 1) clearly shows an effect of stimulus duration, but this effect is small $(<2 \mathrm{~dB})$ and, in the case of the 100 and 500 ms conditions, short lived. An analysis that collapses across a 2100 ms epoch would be susceptible to 'averaging-out' these effects, as was the case.

Second, the initial report restricted analysis of the $\gamma$-band to oscillations under $45 \mathrm{~Hz}$. Task sensitive changes in $\gamma$-power have been shown in frequencies considerably higher than this $45 \mathrm{~Hz}$ upper bound (e.g., Engell and McCarthy, 2010, 2011; Jung et al., 2011; Lachaux et al., 2005; Ray et al., 2008). Here, we investigated frequencies extending up to $100 \mathrm{~Hz}$ and defined the ' $\gamma$-band' as those frequencies between 
30 and $100 \mathrm{~Hz}$. Inspection of the time-frequency plots of the FG electrodes (Fig. 1) reveals why this is a crucial difference. Much of the $\gamma$ power comes from the higher frequencies within this $30-100 \mathrm{~Hz}$ range and would, therefore, be missed by an approach that restricted the analysis to $<45 \mathrm{~Hz}$.

In summary, we find that $\gamma$-ERSP power is consistently coupled with the hemodynamic response, whereas sub-gamma frequencies are not. This finding reconciles the original report with the subsequent literature and demonstrates that the ERP and ERSP reflect dissociable aspects of neural activity.

\section{Acknowledgments}

We thank Dr. Dennis D. Spencer, Dr. Truett Allison, and Mr. Joseph Jasiorkowski for assistance in data acquisition and analysis. This research was supported by National Institute of Neurological Disorders and Stroke grant NS-41328 and by National Institute of Mental Health grant MH-05286.

\section{References}

Delorme, A., Makeig, S., 2004. EEGLAB: an open source toolbox for analysis of singletrial EEG dynamics including independent component analysis. J. Neurosci. Methods 134 (1), 9-21.

DeYoe, E.A., Carman, G.J., Bandettini, P., Glickman, S., Wieser, J., Cox, R., Miller, D., Neitz, J., 1996. Mapping striate and extrastriate visual areas in human cerebral cortex. Proc. Natl. Acad. Sci. U. S. A. 93 (6), 2382-2386.

Engel, S.A., Glover, G.H., Wandell, B.A., 1997. Retinotopic organization in human visual cortex and the spatial precision of functional MRI. Cereb. Cortex 7 (2), 181-192.

Engell, A.D., McCarthy, G., 2010. Selective attention modulates face-specific induced gamma oscillations recorded from ventral occipitotemporal cortex. J. Neurosci. 30 (26), 8780-8786.

Engell, A.D., McCarthy, G., 2011. The relationship of gamma oscillations and facespecific ERPs recorded subdurally from occipitotemporal cortex. Cereb. Cortex 21 (5), 1213-1221.

Fisch, L., Privman, E., Ramot, M., Harel, M., Nir, Y., Kipervasser, S., Andelman, F., Neufeld, M.Y., Kramer, U., Fried, I., Malach, R., 2009. Neural "ignition": enhanced activation linked to perceptual awareness in human ventral stream visual cortex. Neuron 64 (4), 562-574.

Goense, J., Logothetis, N.K., 2008. Neurophysiology of the BOLD fMRI signal in awake monkeys. Curr. Biol. 18 (9), 631-640.

Goldman, R.I., Stern, J.M., Engel Jr., J., Cohen, M.S., 2002. Simultaneous EEG and fMRI of the alpha rhythm. Neuroreport 13 (18), 2487.

Hoge, R.D., Atkinson, J., Gill, B., Crelier, G.R., Marrett, S., Pike, G.B., 1999. Linear coupling between cerebral blood flow and oxygen consumption in activated human cortex. Proc. Natl. Acad. Sci. U. S. A. 96 (16), 9403.

Huettel, S.A., McKeown, M.J., Song, A.W., Hart, S., Spencer, D.D., Allison, T., McCarthy, G., 2004. Linking hemodynamic and electrophysiological measures of brain activity: evidence from functional MRI and intracranial field potentials. Cereb. Cortex 14 (2), 165-173.

Jerbi, J., Ossandón, T., Jung, J., Minotti, M., Bertrand, B., Berthoz, B., Kahane, P., Lachaux, J.P., 2009. Task-related gamma-band dynamics from an intracerebral perspective: review and implications for surface EEG and MEG. Hum. Brain Mapp. 30 (6), 1758-1771.
Jung, J., Bayle, D., Jerbi, K., Vidal, J.R., Hénaff, M.A., Ossandon, T., Bertrand, O., Mauguière, F., Lachaux, J.P., 2011. Intracerebral gamma modulations reveal interaction between emotional processing and action outcome evaluation in the human orbitofrontal cortex. Int. J. Psychophysiol. 79 (1), 64-72.

Kayser, C., Kim, M., Ugurbil, K., Kim, D.S., Konig, P., 2004. A comparison of hemodynamic and neural responses in cat visual cortex using complex stimuli. Cereb. Cortex 14 (8), 881-891.

Koch, S.P., Werner, P., Steinbrink, J., Fries, P., Obrig, H., 2009. Stimulus-induced and state-dependent sustained gamma activity is tightly coupled to the hemodynamic response in humans. J. Neurosci. 29 (44), 13962-13970.

Lachaux, J.P., George, N., Tallon-Baudry, C., Martinerie, J., Hugueville, L., Minotti, L. Kahane, P., Renault, B., 2005. The many faces of the gamma band response to complex visual stimuli. Neuroimage 25 (2), 491-501.

Lachaux, J.P., Fonlupt, P., Kahane, P., Minotti, L., Hoffmann, D., Bertrand, O., Baciu, M. 2007. Relationship between task-related gamma oscillations and BOLD signal: new insights from combined fMRI and intracranial EEG. Hum. Brain Mapp. 28 (12), 1368-1375.

Logothetis, N.K., Pauls, J., Augath, M., Trinath, T., Oeltermann, A., 2001. Neurophysiological investigation of the basis of the fMRI signal. Nature 412 (6843), 150-157.

Malonek, D., Grinvald, A., 1996. Interactions between electrical activity and cortical microcirculation revealed by imaging spectroscopy: implications for functional brain mapping. Science 272 (5261), 551.

Moosmann, M., Ritter, P., Krastel, I., Brink, A., Thees, S., Blankenburg, F., Taskin, B., Obrig, H., Villringer, A., 2003. Correlates of alpha rhythm in functional magnetic resonance imaging and near infrared spectroscopy. Neuroimage 20 (1), $145-158$.

Mukamel, R., Gelbard, H., Arieli, A., Hasson, U., Fried, I., Malach, R., 2005. Coupling between neuronal firing, field potentials, and FMRI in human auditory cortex. Science 309 (5736), 951-954.

Muthukumaraswamy, S.D., Singh, K.D., 2009. Functional decoupling of BOLD and gamma-band amplitudes in human primary visual cortex. Hum. Brain Mapp. 30 (7), 2000-2007.

Niessing, J., Ebisch, B., Schmidt, K.E., Niessing, M., Singer, W., Galuske, R.A.W., 2005. Hemodynamic signals correlate tightly with synchronized gamma oscillations. Science 309 (5736), 948-951.

Ojemann, G.A., Corina, D.P., Corrigan, N., Schoenfield-McNeill, J., Poliakov, A., Zamora, L., Zanos, S., 2010. Neuronal correlates of functional magnetic resonance imaging in human temporal cortex. Brain 133 (1), 46.

Puce, A., Allison, T., Gore, J.C., McCarthy, G., 1995. Face-sensitive regions in human extrastriate cortex studied by functional MRI. J. Neurophysiol. 74 (3), 1192-1199.

Ray, S., Niebur, E., Hsiao, S.S., Sinai, A., Crone, N.E., 2008. High-frequency gamma activity $(80-150 \mathrm{~Hz})$ is increased in human cortex during selective attention. Clin. Neurophysiol. 119 (1), 116-133.

Ritter, P., Moosmann, M., Villringer, A., 2009. Rolandic alpha and beta EEG rhythms' strengths are inversely related to fMRI-BOLD signal in primary somatosensory and motor cortex. Hum. Brain Mapp. 30 (4), 1168-1187.

Sereno, M.I., Dale, A.M., Reppas, J.B., Kwong, K.K., Belliveau, J.W., Brady, T.J., Rosen, B.R., Tootell, R.B., 1995. Borders of multiple visual areas in humans revealed by functional magnetic resonance imaging. Science 268 (5212), 889-893.

Sirotin, Y.B., Das, A., 2009. Anticipatory haemodynamic signals in sensory cortex not predicted by local neuronal activity. Nature 457 (7228), 475-479.

Spencer, S.S., Spencer, D.D., Williamson, P.D., Mattson, R.H., 1982. The localizing value of depth electroencephalography in 32 patients with refractory epilepsy. Ann. Neurol. 12 (3), 248-263.

Viswanathan, A., Freeman, R.D., 2007. Neurometabolic coupling in cerebral cortex reflects synaptic more than spiking activity. Nat. Neurosci. 10 (10), 1308-1312.

Voyvodic, J.T., 1999. Real-time fMRI paradigm control, physiology, and behavior combined with near real-time statistical analysis. Neuroimage 10 (2), 91-106. 